\title{
Orthogonality measures and applications in systems theory in one and more variables
}

\author{
Adhemar Bultheel ${ }^{1}$, Annie Cuyt ${ }^{2}$, and Brigitte Verdonk ${ }^{2}$ \\ 1 Dept of Computer Science, Katholieke Universiteit Leuven, \\ Celestijnenlaan 200A, B3001 Heverlee, Belgium \\ adhemar.bultheel@cs.kuleuven. be \\ 2 Dept of Mathematics and Computer Science, University of Antwerp \\ Middelheimlaan 1, B2020 Antwerp, Belgium \\ \{annie.cuyt, brigitte.verdonk\}@ua.ac.be
}

\begin{abstract}
The representation or order reduction of a rational transfer function by a linear combination of orthogonal rational functions offers several advantages, among which the possibility to work with prescribed poles and hence the guarantee of system stability. Also for multidimensional linear shift-invariant systems with infinite-extent impulse response, stability can be guaranteed a priori by the use of a multivariate Padé-type approximation technique, which is again a rational approximation technique. In both the one- and multidimensional case the choice of the moment functional with respect to which the orthogonality of the functions in use is imposed, plays a crucial role.
\end{abstract}

\section{Introduction}

Let $\left\{c_{i}\right\}_{i \in \mathbb{N}}$ be a sequence of complex numbers and let $c$ be a linear functional defined on the space of polynomials $\mathbb{C}[t]$ with complex coefficients by $c\left(t^{i}\right)=c_{i}$, $i=0,1, \ldots$ Then $c$ is called the moment functional determined by the moment sequence $\left\{c_{i}\right\}_{i \in \mathbb{N}}$. By means of $c$ a formal series development of $h(z)$ with coefficients $c_{i}$ (for instance a transfer function $h(z)$ with impulse response $c_{i}$ for $i=0,1,2, \ldots)$ can be viewed as

$$
h(z)=\sum_{i=0}^{\infty} c_{i} z^{i}=c(1)+c(t) z+c\left(t^{2}\right) z^{2}+\ldots=c\left(\frac{1}{1-t z}\right) .
$$

Let $\mathcal{L}_{n}=\operatorname{span}\left\{1, \ldots, t^{n}\right\}$ denote the space of polynomials of degree $n$ and let $\partial V$ denote the exact degree of a polynomial $V(t) \in \mathbb{C}[t]$. A sequence of polynomials $\left\{V_{m}(z)\right\}_{m \in \mathbb{N}}$ is called orthogonal with respect to the moment functional $c$ provided that $V_{m} \in \mathcal{L}_{m} \backslash \mathcal{L}_{m-1}$ and

$$
c\left(t^{i} V_{m}(t)\right)=0, \quad i=0, \ldots, m-1, \quad c\left(V_{m}^{2}(t)\right) \neq 0 .
$$

For an arbitrary polynomial $V_{m}(z) \in \mathcal{L}_{m}$ with coefficients $b_{i}$, we can also construct the associated polynomial $W_{m-1}(z)$ by

$$
W_{m-1}(z)=c\left(\frac{V_{m}(t)-V_{m}(z)}{t-z}\right) .
$$


Then $W_{m-1} \in \mathcal{L}_{m-1}$ with coefficients $a_{i}=\sum_{j=0}^{m-1-i} c_{j} b_{i+j+1}[3$, p. 10]. It can be proven [3, p. 11] that the polynomials $\tilde{W}_{m-1}(z)=z^{m-1} W_{m-1}(1 / z)$ and $\tilde{V}_{m}(z)=z^{m} V_{m}(1 / z)$ satisfy [3, p. 11]

$$
\left(h-\tilde{W}_{m-1} / \tilde{V}_{m}\right)(z)=\sum_{i=q}^{\infty} d_{i} z^{i}
$$

with $q=m$. In this way it is easy to obtain rational approximants $\tilde{W}_{m-1} / \tilde{V}_{m}$ for a given transfer function $h(z)$. Choosing $V_{m}(z)$ in (4) allows to control the poles of the rational approximant. If however $V_{m}(z)$ is fixed by the orthogonality conditions (2), then $q=2 m$ in (4) and many more moments are matched, but the control over the poles is lost.

We recall that a system is called bounded-input bounded-output (BIBO) stable if the output signal is bounded whenever the input signal is bounded. Since stability is guaranteed when the rational approximant has all its poles inside the unit disk or polydisk, respectively, the aim is to obtain a rational function either in one or in more variables, that has this property.

Rational approximants of higher numerator degree can be obtained in the following way. If we define a linear functional $c^{(k)}\left(t^{i}\right)=c_{k+i}$ and set

$$
\begin{aligned}
h(z) & =\sum_{i=0}^{k} c_{i} z^{i}+z^{k+1} h_{k}(z), \\
W_{m-1}^{(k+1)}(z) & =c^{(k+1)}\left(\frac{V_{m}(t)-V_{m}(z)}{t-z}\right) \\
\tilde{W}_{m+k}(z) / \tilde{V}_{m}(z) & =\sum_{i=0}^{k} c_{i} z^{i}+z^{k+1} \tilde{W}_{m-1}^{(k+1)}(z) / \tilde{V}_{m-1}(z)
\end{aligned}
$$

then (4) generalizes to

$$
\left(h-\tilde{W}_{m+k} / \tilde{V}_{m}\right)(z)=\sum_{i=q}^{\infty} d_{i} z^{i}
$$

with $q=m+k+1$ for arbitrary polynomials $V_{m}(z)$ and $q=2 m+k+1$ when $V_{m}(z)$ satisfies the orthogonality conditions (2).

In (1)-(4), a linear functional is defined in terms of the impulse response, and the rational function that approximates the transfer function matches as many of the initial impulse response coefficients, also called Markov parameters, as possible. This corresponds to a good approximation of the transient behaviour of the system for small time. In this paper we discuss the generalization of the steps (1)-(4), namely

- defining a linear functional $c$ using information collected from the transfer function $h$ as in (1),

- computing a numerator polynomial $\tilde{W}_{m-1}(z)$ as in (3), possibly in combination with (2) for the denominator polynomial $\tilde{V}_{m}(z)$, 
- setting up a sequence of rational approximants to the transfer function $h(z)$ as in (4) or (8),

in two ways.

The approximation of $h(z)$ in (4) may be improved when also the steady state of the system is approximated. This means that the transfer function is not only approximated in the neighborhood of $z=0$, but also near $z=\infty$. The coefficients of the series expansion at infinity are called time moments. Matching some of the Markov parameters and some of the time moments corresponds to rational approximation in two points. This idea can be generalized as follows. Instead of approximating in just two points, one can find a rational approximant interpolating in several points, some of which may coincide [5].

Instead of considering one variable $z$, one can also study multidimensional systems and transfer functions, which arise in problems like computer-aided tomography, image processing, image deblurring, seismology, sonar and radar applications, and many other problems. As an illustration we consider the filtering of signals, which is concerned with the extraction and/or enhancement of information contained in either a one-dimensional or multidimensional sequence of measurements. Noises can be filtered from spoken messages as well as from picture images.

The former generalization is dealt with in Section 2 while the latter is introduced in Section 3. In both sections the aim is to provide an a priori stable rational approximant since for model reduction techniques the issue of stability of the reduced rational system is an important one. In Section 2 the rational approximants are obtained by combining generalizations of (3) and (2), while in Section 3 the approximants are constructed for appropriately chosen denominator polynomials in combination with (3) for the numerator.

\section{Orthogonal rational functions analytic outside the unit disk}

In frequency domain methods, it is assumed that the information about the system transfer function is not given by moments defined in 0 and infinity or at arbitrary points in the complex plane, but they are given in the frequency domain, which for a discrete time system is the complex unit circle $\mathbb{T}$. So what can be measured are not the samples of the transfer function $h$, but samples of its power spectrum $|h(z)|^{2}$ for many values of $z \in \mathbb{T}$. Using autocorrelation techniques, one actually knows the coefficients of the Fourier series $|h(z)|^{2}=$ $\sum_{k \in \mathbb{Z}} c_{k} z^{k}$ where $z=e^{\mathrm{i} \omega}$. We can now define a moment functional for the Laurent polynomials by setting $c\left(t^{i}\right)=c_{-i}$ for all $i \in \mathbb{Z}$. Since we are working on $\mathbb{T}$ we reformulate the orthogonality conditions $(2)$ as follows. A sequence of polynomials $\left\{V_{m}(z)\right\}_{m \in \mathbb{N}}$ is orthogonal with respect to the moment functional $c$ provided that $V_{m}(z) \in \mathcal{L}_{m} \backslash \mathcal{L}_{m-1}$ and

$$
c\left(t^{i} V_{m *}(t)\right)=0, \quad i=0, \ldots, m-1, \quad c\left(V_{m}(t) V_{m *}(t)\right) \neq 0,
$$


where for any function $f$ we define $f_{*}(z)=\overline{f(1 / \bar{z})} .^{3}$

Knowing $c$, the problem is still to approximate $h$. The original approach $[9,8]$ is to construct an autoregressive (AR) approximant, i.e., one of the form $R_{m}(z)=K z^{m} / \tilde{V}_{m}(z)$ with $K$ a constant and $V_{m}$ the orthogonal polynomial with respect to $c$. A relation similar to (4) can be derived, not for the transfer function $h$ but for "half the Fourier series" $\Omega(z)=c_{0}+2 \sum_{k>0} c_{k} z^{k}$.

We now give a generalization that results in an approximant $R_{m}$ with a nonconstant numerator (ARMA model). Consider a sequence $\alpha_{k}$ with $\left|\alpha_{k}\right|<1$ for all $k$. The classical AR case will pop up as the special situation where all $\alpha_{k}=$ 0 . The $\alpha_{k}$ will turn out to play a multiple role (i) as the zeros of the approximant $R_{m}$, (ii) as the reciprocals of the poles of the orthogonal rational functions (which generalize the orthogonal polynomials) and (iii) as the interpolation points for the multipoint version of the approximation (4) to $\Omega$.

Consider the kernel $D(t, z)=\frac{(t+z)}{(t-z)}$ with formal expansion [4, p. 240]

$$
D(t, z)=1+2 \sum_{k=1}^{\infty} a_{k}(t) z \pi_{k-1}(z), \quad a_{k}(t)=\frac{1}{\pi_{k}(t)}, \quad \pi_{k}(z)=\prod_{i=1}^{k}\left(z-\alpha_{i}\right) .
$$

Then, assuming for simplicity of notation but without loss of generality, that $\int_{-\pi}^{\pi}\left|h\left(e^{i \theta}\right)\right|^{2} d \theta=1$, we get, at least formally,

$$
\begin{aligned}
\Omega(z) & =\int_{-\pi}^{\pi} D\left(e^{i \theta}, z\right)\left|h\left(e^{\mathrm{i} \theta}\right)\right|^{2} d \theta=c_{0}+2 \sum_{k=1}^{\infty} c_{k} z \pi_{k-1}(z), \\
c_{0} & =1, \quad c_{k}=\int_{-\pi}^{\pi} a_{k}\left(e^{\mathrm{i} \theta}\right)\left|h\left(e^{\mathrm{i} \theta}\right)\right|^{2} d \theta, \quad k=1,2, \ldots
\end{aligned}
$$

Observe that if all $\alpha_{k}=0$, then $\pi_{k}(z)=z^{k}$, and the $c_{k}$ are the trigonometric moments, in other words the Fourier coefficients of $|h(z)|^{2}$ for $z \in \mathbb{T}$. Since the definition of $\Omega$ does not depend on the choice of the $\alpha_{k}$, we can see that $\Omega(z)$ is the same as introduced above. It is an analytic function in $|z|<1$.

For general prefixed $\alpha_{k}$, let $a_{k}(t)$ be given by (10) and $c_{k}$ be given by (11). One can define a linear functional $c$ on the space $\mathcal{L}=\operatorname{span}\left\{1, a_{1}(t), a_{2}(t), \ldots\right\}$ via $c\left(a_{k}\right)=c_{k}, k=0,1,2, \ldots$ For negative $k$, we set $a_{-k}=a_{k *}$, so that $c_{-k}=$ $\bar{c}_{k}$, and using partial fraction expansion, we may even assume that the linear functional $c$ is defined on $\mathcal{L} \cdot \mathcal{L}_{*}=\mathcal{L}+\mathcal{L}_{*}$ where $\mathcal{L}_{*}=\left\{f: f_{*} \in \mathcal{L}\right\}$ by the relation $c\left(a_{k}\right)=c_{k}, k \in \mathbb{Z}$.

The sequence of orthogonal polynomials becomes a sequence of orthogonal rational functions with polynomials as a special case. A sequence of rational functions $V_{m}(z) \in \mathcal{L}_{m}=\operatorname{span}\left\{1, a_{1}(z), \ldots, a_{m}(z)\right\}$ is called orthogonal with respect to the moment functional $c$ defined on $\mathcal{L} \cdot \mathcal{L}_{*}$ as outlined above, provided that $V_{m} \in \mathcal{L}_{m} \backslash \mathcal{L}_{m-1}$ and that the rational functions $V_{m}$ satisfy the relations (9) with $t^{i}$ replaced by the rational basis $a_{i}(t)=1 / \pi_{i}(t)$ from (10). It turns out

\footnotetext{
${ }^{3}$ Observe that for $t \in \mathbb{T}, f_{*}(t)=\overline{f(t)}$.
} 
that for $m \geq 1$, the associated functions $W_{m}$ defined by

$$
W_{m}(z)=c\left(D(t, z)\left(V_{m}(t)-V_{m}(z)\right)\right)
$$

also belong to $\mathcal{L}_{m}[4$, Eq. (4.21)].

Moreover one has interpolation properties of the following type [4, Theorem 6.1.4]

$$
\begin{aligned}
& \Omega(z)-\frac{W_{m}(z)}{V_{m}(z)}=z \pi_{m}(z) g_{+}(z), \quad g_{+} \text {analytic in }|z|<1 \\
& \Omega(z)-\frac{W_{m *}(z)}{V_{m *}(z)}=\left(z \pi_{m}(z)\right)_{*} g_{-}(z), \quad g_{-} \text {analytic in }|z|>1, \text { including } \infty .
\end{aligned}
$$

The ratios are well defined because the linear functional is positive definite, which implies that the zeros of $V_{m}$ are all in $|z|>1$ and hence, the zeros of $V_{m *}$ are all in $|z|<1$. In case all $\alpha_{k}=0$ (the polynomial or AR case), we match the first $m$ coefficients of the series expansion of $\Omega$ in $z=0$ and in $z=\infty$ respectively. In general, as the above interpolation properties show, the AR interpolation conditions in $z=0$ are replaced by ARMA interpolation conditions in $z=0, \alpha_{1}, \ldots, \alpha_{m}$ and the AR interpolation conditions in $z=\infty$ are distributed over the points $z=\infty, 1 / \bar{\alpha}_{1}, \ldots, 1 / \bar{\alpha}_{m}$ taking multiplicity into account. This is multipoint moment matching.

To come to the original problem of approximating $h$ itself, one makes use of the determinant formula [4, Theorem 4.2.6]

$$
\frac{1}{2}\left(W_{m}(z) V_{m *}(z)+W_{m *}(z) V_{m}(z)\right)=\frac{1-\left|\alpha_{m}\right|^{2}}{\left(1 / z-\bar{\alpha}_{m}\right)\left(z-\alpha_{m}\right)} .
$$

Recall that for $z \in \mathbb{T}, \Omega(z)$ is the real part of $|h(z)|^{2}$, in other words $\left|h\left(e^{\mathrm{i} \omega}\right)\right|^{2}=$ $\frac{1}{2}\left(\Omega\left(e^{\mathrm{i} \omega}\right)+\Omega_{*}\left(e^{\mathrm{i} \omega}\right)\right)$. It then follows, after dividing the previous relation for $z=e^{\mathrm{i} \omega}$ by $V_{m}(z) V_{m *}(z)=\left|V_{m}(z)\right|^{2}$, that

$$
\left|h\left(e^{\mathrm{i} \omega}\right)\right|^{2} \approx\left|\frac{K}{\left(e^{\mathrm{i} \omega}-\alpha_{m}\right) V_{m}\left(e^{\mathrm{i} \omega}\right)}\right|^{2}=\left|\frac{K \pi_{m-1}\left(e^{\mathrm{i} \omega}\right)}{P_{m}\left(e^{\mathrm{i} \omega}\right)}\right|^{2}, \quad V_{m}\left(e^{\mathrm{i} \omega}\right)=\frac{P_{m}\left(e^{\mathrm{i} \omega}\right)}{\pi_{m}\left(e^{\mathrm{i} \omega}\right)},
$$

with $K=\sqrt{1-\left|\alpha_{m}\right|^{2}}$. Knowing that $h$ is analytic in $|z|>1$ if the system is stable, we can approximate it by $K \pi_{m-1} / P_{m}$ as described above. Note that the $\alpha_{k}$ which are chosen as the poles of space $\mathcal{L}_{m}$ of rational functions are interpolation points when approximating $\Omega$ and that they now show up as the zeros of the approximating transfer function.

\section{Homogeneous Padé-type approximants analytic outside the unit polydisk}

To deal with multivariate polynomials and functions we switch between the cartesian and the spherical coordinate system. The cartesian coordinates $X=$ 
$\left(x_{1}, \ldots, x_{n}\right) \in \mathbb{C}^{n}$ are then replaced by $X=\left(\xi_{1} z, \ldots, \xi_{n} z\right)$ with $\xi_{k} \in \mathbb{C}, z \in \mathbb{R}$ where the directional vector $\xi=\left(\xi_{1}, \ldots, \xi_{n}\right)$ belongs to the unit sphere $S_{n}=$ $\left\{\xi:\|\xi\|_{p}=1\right\}$. Here $\|\cdot\|_{p}$ denotes one of the usual Minkowski norms. While $\xi$ contains the directional information of $X$, the variable $z$ contains the (possibly signed) distance information. With the multi-index $\kappa=\left(\kappa_{1}, \ldots, \kappa_{n}\right) \in \mathbb{N}^{n}$ the notations $X^{\kappa}, \kappa$ ! and $|\kappa|$ respectively denote

$$
\begin{aligned}
X^{\kappa} & =x_{1}^{\kappa_{1}} \ldots x_{n}^{\kappa_{n}}, \\
\kappa ! & =\kappa_{1} ! \ldots \kappa_{n} ! \\
|\kappa| & =\kappa_{1}+\ldots+\kappa_{n},
\end{aligned}
$$

and with $X$, we associate its signed distance

$$
\operatorname{sd}(X)=\operatorname{sgn}\left(x_{1}\right)\|X\|_{p} .
$$

Note that it is always possible to choose $\xi$ such that $z=\operatorname{sd}(X)$. For the sequel of the discussion we need some more notation. We denote by $\mathbb{C}[\xi]:=\mathbb{C}\left[\xi_{1}, \ldots, \xi_{n}\right]$ the linear space of $n$-variate polynomials in $\xi_{k}$ with complex coefficients, by $\mathbb{C}(\xi):=\mathbb{C}\left(\xi_{1}, \ldots, \xi_{n}\right)$ the commutative field of rational functions in $\xi_{k}$ with complex coefficients, by $\mathbb{C}(\xi)[z]$ the linear space of polynomials in the variable $z$ with coefficients from $\mathbb{C}(\xi)$ and by $\mathbb{C}[\xi][z]$ the linear space of polynomials in the variable $z$ with coefficients from $\mathbb{C}[\xi]$.

Let us introduce the linear functional $C$ acting on the signed distance variable $z$ as $C\left(z^{i}\right)=c_{i}(\xi)$, where $c_{i}(\xi)$ is a homogeneous expression of degree $i$ in the $\xi_{k}: c_{i}(\xi)=\sum_{|\kappa|=i} c_{\kappa} \xi^{\kappa}$. Then $C$ is a multivariate moment functional with multidimensional moments $c_{\kappa}$. Multivariate orthogonality with respect to the linear functional $C$ can be defined [6]. The $n$-variate polynomials under investigation here, are of the form

$$
V_{m}(X)=\mathcal{V}_{m}(z)=\sum_{i=0}^{m} B_{m-i}(\xi) z^{i} \quad B_{m-i}(\xi)=\sum_{|\kappa|=m-i} b_{\kappa} \xi^{\kappa} .
$$

The function $V_{m}(X)$ is a polynomial of degree $m$ in $z$ with polynomial coefficients from $\mathbb{C}[\xi]$. The coefficients $B_{0}(\xi), \ldots, B_{m}(\xi)$ are homogeneous polynomials in the parameters $\xi_{k}$. The function $V_{m}(X)$ does itself not belong to $\mathbb{C}[X]$, but as $V_{m}(X)$ can be viewed as $\mathcal{V}_{m}(z)$, it belongs to $\mathbb{C}[\xi][z]$. Therefore the function $V_{m}(X)$ can be coined a spherical polynomial: for every $\xi \in S_{n}$ we can identify the function $V_{m}(X)$ with the polynomial $\mathcal{V}_{m}(z)$ of degree $m$ in the variable $z=\operatorname{sd}(X)$.

With an arbitrarily chosen $V_{m}(X)$ we can associate the function $W_{m-1}(X)$ defined by

$$
W_{m-1}(X)=\mathcal{W}_{m-1}(z)=C\left(\frac{\mathcal{V}_{m}(t)-\mathcal{V}_{m}(z)}{t-z}\right) .
$$

One can show [2] that $W_{m-1}(X)$ is a polynomial of degree $m-1$ in $z$, but not that it is a polynomial in $X$. Instead it belongs to $\mathbb{C}[\xi][z]$ and has the form

$$
W_{m-1}(X)=\mathcal{W}_{m-1}(z)=\sum_{i=0}^{m-1} A_{m-1-i}(\xi) z^{i}
$$


For the polynomials $\tilde{V}_{m}(X)$ and $\tilde{W}_{m-1}(X)$ defined by

$$
\begin{gathered}
\tilde{V}_{m}(X)=\tilde{\mathcal{V}}_{m}(z)=z^{m} \mathcal{V}_{m}\left(z^{-1}\right)=\sum_{k=0}^{m} \tilde{B}_{k}(\xi) z^{k}=\sum_{k=0}^{m}\left(\sum_{|\kappa|=k} \tilde{b}_{\kappa} X^{\kappa}\right) \\
\tilde{W}_{m-1}(X)=\tilde{\mathcal{W}}_{m-1}(z)=z^{m-1} \mathcal{W}_{m-1}\left(z^{-1}\right)=\sum_{k=0}^{m-1} \tilde{A}_{k}(\xi) z^{k}=\sum_{k=0}^{m-1}\left(\sum_{|\kappa|=k} \tilde{a}_{\kappa} X^{\kappa}\right)
\end{gathered}
$$

and belonging to $\mathbb{C}[X]$, it can be proved that $[2]$

$$
\left(f \tilde{V}_{m}-\tilde{W}_{m-1}\right)(X)=\left(f \tilde{\mathcal{V}}_{m}-\tilde{\mathcal{V}}_{m-1}\right)(z)=\sum_{i=m}^{\infty} d_{i}(\xi) z^{i}=\sum_{i=m}^{\infty}\left(\sum_{|\kappa|=i} d_{\kappa} X^{\kappa}\right) .
$$

As in (5)-(8), the linear functional $C$ and the rational function $\tilde{W}_{m-1} / \tilde{V}_{m}(X)$ can be generalized to $C^{(k)}$ and $\tilde{W}_{m+k} / \tilde{V}_{m}(X)$.

Now let us consider a multidimensional LSI system with IIR [7] and transfer function $H(X)=F(Y) / G(Y)$ where $F(Y)$ and $G(Y)$ are polynomials in the variables $y_{i}=x_{i}^{-1}$ and $Y=\left(y_{1}, \ldots, y_{n}\right)$. In terms of the impulse response $c_{\kappa}$ (without loss of generality we restrict ourselves to support on the first quadrant), we have:

$$
H(X)=\sum_{|\kappa|=0}^{\infty} c_{\kappa} Y^{\kappa} .
$$

The system is stable if $G(Y)$ has all its zeroes strictly inside the unit polydisc. A stable identification or model order reduction of $H(X)$ can be given by $\tilde{W}_{m+k}(Y) / \tilde{V}_{m}(Y)$ provided $\tilde{V}_{m}(Y)$ has all its zeroes inside the unit polydisc [1].

Let us illustrate the above in the context of IIR filter design $(n=2)$. An ideal lowpass filter can be specified by the frequency response $\left(x_{1}=\exp \left(\mathrm{it} t_{1}\right), x_{2}=\right.$ $\left.\exp \left(\mathrm{i} t_{2}\right)\right)$

$$
H\left(e^{\mathrm{i} t_{1}}, e^{\mathrm{i} t_{2}}\right)= \begin{cases}1 & \left(t_{1}, t_{2}\right) \in T \subset[-\pi, \pi] \times[-\pi, \pi] \\ 0 & \left(t_{1}, t_{2}\right) \notin T\end{cases}
$$

where $T$ is usually a symmetric domain. For $T=[-\pi / 8, \pi / 8] \times[-\pi / 8, \pi / 8]$ we have for instance

$$
c_{\kappa_{1}, \kappa_{2}}=\frac{\sin \left(\frac{\pi}{8} \kappa_{1}\right)}{\pi \kappa_{1}} \frac{\sin \left(\frac{\pi}{8} \kappa_{2}\right)}{\pi \kappa_{2}} .
$$

Let us in addition impose the quadrant symmetry conditions

$$
\begin{aligned}
\frac{\tilde{W}_{m+k}}{\tilde{V}_{m}}\left(e^{\mathrm{i} t_{1}}, e^{\mathrm{i} t_{2}}\right) & =\frac{\tilde{W}_{m+k}}{\tilde{V}_{m}}\left(e^{-\mathrm{i} t_{1}}, e^{\mathrm{i} t_{2}}\right)=\frac{\tilde{W}_{m+k}}{\tilde{V}_{m}}\left(e^{\mathrm{i} t_{1}}, e^{-\mathrm{i} t_{2}}\right) \\
& =\frac{\tilde{W}_{m+k}}{\tilde{V}_{m}}\left(e^{-\mathrm{i} t_{1}}, e^{-\mathrm{i} t_{2}}\right) .
\end{aligned}
$$

Then with the choice

$$
V_{2}(Y)=1.94145 z^{2}-1.30911\left(\xi_{1}+\xi_{2}\right) z+0.340194\left(\xi_{1}^{2}+\xi_{2}^{2}\right)+0.000033 \xi_{1} \xi_{2}
$$


which guarantees a stable filter, we find

$$
\frac{\tilde{W}_{2}(Y)}{\tilde{V}_{2}(Y)}=\frac{303.351+91.0655\left(y_{1}+y_{2}\right)+126.936\left(y_{1}^{2}+y_{2}^{2}\right)-110.584 y_{1} y_{2}}{358.005\left(1.94145-1.30911\left(y_{1}+y_{2}\right)+0.340194\left(y_{1}^{2}+y_{2}^{2}\right)+0.000033 y_{1} y_{2}\right)}
$$

The frequency response $\tilde{W}_{2}(Y) / \tilde{V}_{2}(Y)$ is shown in Figure 3.a. The contour lines $\left|\left(\tilde{W}_{2} / \tilde{V}_{2}\right)\left(e^{\mathrm{it} t_{1}}, e^{\mathrm{it} t_{2}}\right)\right|=0.1$ and $\left|\left(\tilde{W}_{2} / \tilde{V}_{2}\right)\left(e^{\mathrm{i} t_{1}}, e^{\mathrm{it} t_{2}}\right)\right|=0.5$ are shown in Figure 3.b.
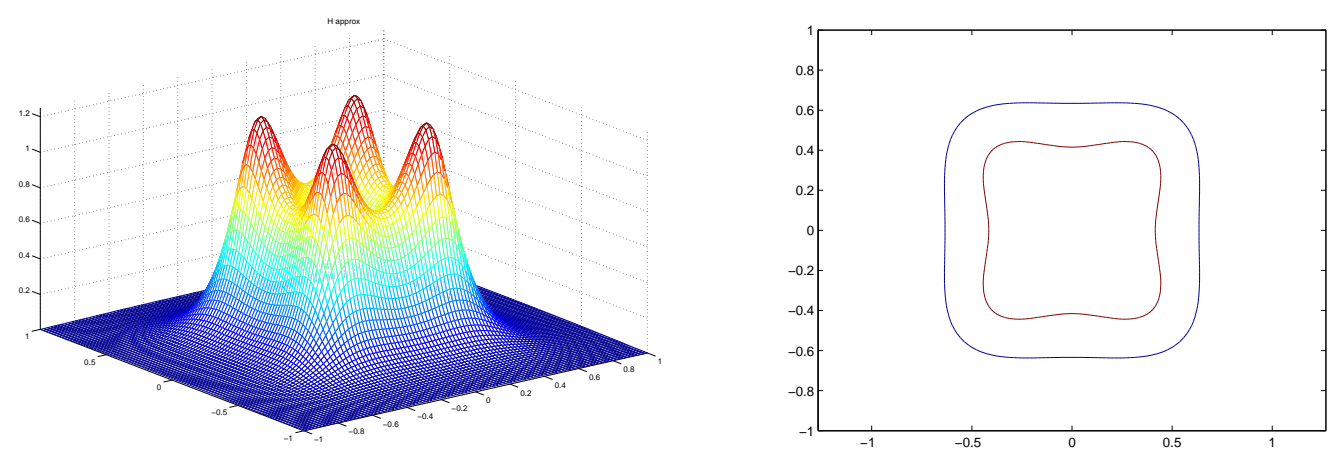

\section{References}

1. J. Abouir and A. Cuyt. Stable multi-dimensional model reduction and IIR filter design. Int. J. Computing Science and Mathematics, 1(1):16-27, 2007.

2. B. Benouahmane and A. Cuyt. Properties of multivariate homogeneous orthogonal polynomials. J. Approx. Theory, 113:1-20, 2001.

3. C. Brezinski. Padé type approximation and general orthogonal polynomials. ISNM 50, Birkhauser Verlag, Basel, 1980.

4. A. Bultheel, P. González-Vera, E. Hendriksen, and O. Njåstad. Orthogonal rational functions, volume 5 of Cambridge Monographs on Applied and Computational Mathematics. Cambridge University Press, 1999.

5. A. Bultheel and M. Van Barel. Padé techniques for model reduction in linear system theory. J. Comput. Appl. Math., 14:401-438, 1986.

6. A. Cuyt, B. Benouahmane, and B. Verdonk. Spherical orthogonal polynomials and symbolic-numeric Gaussian cubature formulas. In M.et al. Bubak, editor, LNCS 3037, pages 557-560, Berlin, 2004. Springer-Verlag.

7. D. Dudgeon and R. Mersereau. Multidimensional digital signal processing. Prentice Hall, Englewood Cliffs, 1984.

8. U. Grenander and G. Szegő. Toeplitz forms and their applications. University of California Press, Berkley, 1958.

9. N. Wiener. Extrapolation, interpolation and smoothing of time series. Wiley, New York, 1949. 